\title{
Pregnancy duration and endometrial cancer risk: nationwide cohort study
}

\author{
Anders Husby, ${ }^{1,2}$ Jan Wohlfahrt, ${ }^{1}$ Mads Melbye ${ }^{1,3,4}$
}

${ }^{1}$ Department of Epidemiology

Research, Statens Serum

Institut, Artillerivej 5, DK-2300

Copenhagen, Denmark

${ }^{2}$ Department of Biomedical

Data Science, Stanford

University School of Medicine,

Stanford, CA USA

${ }^{3}$ Department of Clinical

Medicine, University of

Copenhagen, Copenhagen,

Denmark

${ }^{4}$ Department of Medicine, Stanford University School of Medicine, Stanford, CA, USA

Correspondence to: A Husby andh@ssi.dk

(or@a_husby on Twitter;

ORCID 0000-0002-7634-8455)

Additional material is published online only. To view please visit

the journal online.

Cite this as: BMJ 2019;366:14693 http://dx.doi.org/10.1136/bmj.14693

Accepted: 4 July 2019

\section{ABSTRACT}

OBJECTIVE

To explore the association between pregnancy

duration and risk of endometrial cancer.

DESIGN

Nationwide register based cohort study.

SETTING

Denmark.

\section{PARTICIPANTS}

All Danish women born from 1935 to 2002.

\section{MAIN OUTCOME MEASURES}

Relative risk (incidence rate ratio) of endometrial cancer by pregnancy number, type, and duration, estimated using log-linear Poisson regression.

\section{RESULTS}

Among 2311332 Danish women with 3947650 pregnancies, 6743 women developed endometrial cancer during 57347622 person years of follow-up. After adjustment for age, period, and socioeconomic factors, a first pregnancy was associated with a noticeably reduced risk of endometrial cancer, whether it ended in induced abortion (adjusted relative risk 0.53 (95\% confidence interval 0.45 to 0.64$)$ or childbirth $(0.66,0.61$ to 0.72$)$. Each subsequent pregnancy was associated with an additional reduction in risk, whether it ended in induced abortion $(0.81,0.77$ to 0.86$)$ or childbirth $(0.86,0.84$ to 0.89$)$. Duration of pregnancy, age at pregnancy, spontaneous abortions, obesity, maternal birth cohort, fecundity, and socioeconomic factors did not modify the results.

\section{CONCLUSIONS}

The risk of endometrial cancer is reduced regardless of whether a pregnancy ends shortly after conception or at 40 weeks of gestation. This reduction in risk could be explained by a biological process occurring within the first weeks of pregnancy, as pregnancies ending in induced abortions were associated with similar reductions in risk as pregnancies ending in childbirth.

\section{WHAT IS ALREADY KNOWN ON THIS TOPIC}

The lifetime number of births is associated with a reduction in risk of endometrial cancer

The number of menstrual cycles suppressed by pregnancy has been suggested to be protective

\section{WHAT THIS STUDY ADDS}

This study found that the risk of endometrial cancer is reduced regardless of whether a woman's pregnancy ends shortly after conception or at 40 weeks This finding strongly suggests that factors early in gestation are responsible for the reduction in risk

\section{Introduction}

Endometrial cancer is the most common gynaecological cancer in developed countries, with an increasing incidence in North America and Europe. ${ }^{12}$ A woman's risk of endometrial cancer has been strongly associated with number of full term pregnancies, with a noticeable protection associated with the first full term pregnancy and additional protection associated with subsequent full term pregnancies. ${ }^{3-12}$ To date studies have focused little on the effect of the duration of pregnancy, and in particular shorter pregnancies (eg, induced abortions and preterm childbirths), in analysis of this protective factor. ${ }^{5}$ It is therefore unknown whether the protective association is driven by the ability to conceive (a woman's fecundity), the cumulative number of months pregnant, or a process that occurs at a specific time during pregnancy.

We combined data from the nationwide Danish National Registry of Induced Abortions, the Medical Birth Registry, and the Danish Cancer Registry to investigate the association between pregnancy duration and risk of endometrial cancer. The nationwide cohort comprised 2.3 million women followed from January 1978 to December 2014 who had more than 670000 induced abortions and 3.2 million births.

\section{Methods}

\section{Population cohort}

Since 1 April 1968 the Danish Civil Registration System has assigned a unique identification number to all Danish residents who were alive on that date or born thereafter. In addition, the register contains detailed personal information on all Danish residents, including linkage of women to their children's dates of birth. Using the unique identification numbers, we created a population based cohort of all Danish women and their pregnancies by linking data from the Civil Registration System with data from the National Registry of Induced Abortions and the Medical Birth Registry.

\section{Study variables}

Since 1939 it has been mandatory in Denmark to report information on induced abortions to the National Registry of Induced Abortions, including the date of the procedure and the week of gestation. Induced abortions were only permitted for medical or other specified reasons until 1973. Thereafter, induced abortion until the end of the 12th week of gestation became legalised. Childbirths in Denmark, including dates of birth, have been registered since 1973 in the Medical Birth Registry, and gestational week at time of birth has been recorded from 1978 .

From Statistics Denmark we acquired time varying, individual level socioeconomic data to address factors 
potentially associated with reproductive choices and cancer risk: educational attainment, marital status, and urbanicity (see supplementary appendix).

From the Danish National Patient Registry we acquired information on hospital diagnostic codes for obesity and non-malignant endometrial and ovarian diseases. We also obtained information on hysterectomies and bilateral oophorectomies, as registered by the Danish Classification of Surgical Procedures and Therapies from 1977 to 1995 and by the Nordic Medico-Statistical Committee Classification of Surgical Procedures from 1996 (see supplementary appendix). The Danish National Patient Registry also holds information on spontaneous abortions that resulted in clinical contact ${ }^{13}$ from 1977, but the registry does not hold detailed information on gestational week for these pregnancies.

Information on endometrial cancer and other cancer diagnoses was collected from the Danish Cancer Registry, which contains details on cancers diagnosed in Denmark since 1943 and is considered close to complete. ${ }^{14}$ Endometrial cancer was defined by ICD-10 (international classification of diseases, 10th revision) codes C54-55 in combination with an endometrial cancer histological subtype by relevant ICD-0-3 (international classification of diseases for oncology, third edition) code (see supplementary appendix). Information on cancer stage (grouped using FIGO (International Federation of Gynecology and Obstetrics) classification ${ }^{15}$ ) and cancer subtype (by previously used grouping of subtype histology ${ }^{16}$ ) was also retrieved from the Danish Cancer Registry.

\section{Participants}

We established a cohort of all Danish women born between 1 January 1935 and 31 December 2002. Using the Danish Civil Registration System identification number, we linked information on each woman's pregnancies with the corresponding pregnancy type (ie, gestational week of every induced abortion) and duration (gestational week of delivery for every birth), and information on whether she developed endometrial cancer. Women were followed from 1 January 1978, or from their 12th birthday, whichever came later, until endometrial cancer, death, emigration, or 31 December 2014, whichever came first. We censored on cancer before start of follow-up, first diagnosis of any other cancer (excluding non-melanoma skin cancer), time of hysterectomy (including hystero-oophorectomy), and time of bilateral oophorectomy.

\section{Statistical analyses}

We used log-linear Poisson regression to estimate incidence rate ratios (relative risks) of endometrial cancer by pregnancy type. Analyses were adjusted for effects of attained age and time in five year categories, in addition to interaction between age and time. Analyses, except when otherwise stated, were adjusted for pregnancy history, educational attainment, marital status, and urbanicity, in addition to interaction between time and the three socioeconomic variables (see supplementary appendix).

Pregnancy history was modelled as described previously, ${ }^{17}$ with the effect of each pregnancy estimated as the effect of the pregnancy compared with one pregnancy less (ie, relative risk of endometrial cancer for one pregnancy compared with 0 , two compared with one, three compared with two, and so on), where a pregnancy could result in either an induced abortion or birth. This parameterisation allows for a focus on the effect of each additional pregnancy. In additional analyses, we assumed relative risks to be the same for all pregnancies after the first pregnancy. To investigate whether the effect of induced abortions and childbirths varied by pregnancy duration $(\leq 5$ weeks, 6-7 weeks, 8-9 weeks, 10-11 weeks, $\geq 12$ weeks for induced abortions, and $<37$ weeks, $\geq 37$ weeks for childbirths), age at pregnancy ( $<30$ years, $\geq 30$ years), time since pregnancy ( $<10$ years, $\geq 10$ years), and attained age ( $<50$ years, $\geq 50$ years), we allowed the relative risks to vary by these factors.

In sensitivity analyses we also considered potential confounding effects of obesity and oral contraceptives use. For this we investigated the effects of pregnancy on risk of endometrial cancer in subcohorts by clinical diagnosis of obesity and birth cohort, respectively.

Additionally, to investigate whether the effects of induced abortions and births were modified by previous induced abortions and births, we analysed the relative risk of endometrial cancer among parous women stratified by the number of induced abortions $(0,1$, or $\geq 2$ ) and births (1, 2, 3, or $\geq 4)$. Uniparous women with no induced abortions were used as reference.

Finally, to explore potential effects of fecundity, we estimated the relative risk of endometrial cancer after a pregnancy (including both induced abortions and births) compared with one pregnancy less by age at and time since any pregnancy. In these analyses, age at pregnancy was stratified by less than 25 years, 25-29 years, 30-34 years, and 35 or more years, whereas time since pregnancy was stratified by less than 10 years, 10-19 years, 20-29 years, and 30 or more years. In addition, we created a high fecundity subcohort, which was defined as women with three or more pregnancies and a maximum of five years between their first and third pregnancy.

All tests were likelihood ratio tests. Analyses were performed using SAS procedure GENMOD.

\section{Patient and public involvement}

No patients were involved in setting the research question, outcome measures, the study design, or the conduct of the study. The results of the study will be disseminated to the public, patients, and health professionals by various media sources: press releases written using layman's terms, social media postings, and scientific conferences.

\section{Results}

The cohort included 2311332 Danish women who were followed for 57347622 person years (average 
of 24.8 years of follow-up per woman). The women had 3947650 pregnancies, of which 671560 were induced abortions and 3276090 were births. The median gestational weeks of pregnancies that ended in induced abortion was 8 (interquartile range 7-9 weeks), whereas the median gestational weeks of pregnancies that ended in birth was 40 (39-41 weeks). During follow-up, 6743 women developed endometrial cancer. Supplementary table S1 shows the number of endometrial cancer events and person years according to number of induced abortions, number of births, age at first pregnancy, age at latest pregnancy, duration of latest pregnancy, time since latest pregnancy, birth cohort, and socioeconomic factors.

Table 1 shows the relative risk of endometrial cancer after any pregnancy compared with one pregnancy less by type of pregnancy. We found that both induced abortions and births were associated with a reduced risk of endometrial cancer, with a particularly strong risk reduction after the first pregnancy (relative risk reduction around 40\%). For the first pregnancy, an induced abortion was associated with a slightly larger risk reduction than a birth, but for subsequent pregnancies the association did not differ $(\mathrm{P}=0.25)$. However, for pregnancies taking place in the same period (from 1973 when induced abortion was legalised in Denmark), induced abortions and births were associated with the same risk reduction in endometrial cancer for both the first $(\mathrm{P}=0.50)$ and any subsequent pregnancy $(\mathrm{P}=0.41)$ (see supplementary table S2). The pattern was the same when stratifying further by duration of pregnancy (table 2).

When stratifying by age at pregnancy (table 3), no effect modification was found on the association between pregnancy type and endometrial cancer
$(\mathrm{P}=0.69)$, when stratifying by time since pregnancy (table 3), no effect modification was found on the association between pregnancy type and endometrial cancer $(\mathrm{P}=0.94)$, and when stratifying by attained age (table 3), no effect modification was found on the association between pregnancy type and endometrial cancer $(\mathrm{P}=0.13)$. In addition, we performed analyses of the effect of pregnancy type on risk of endometrial cancer by cancer stage and subtype (table 4), by adjustment for non-malignant endometrial and ovarian disease (see supplementary table S3), and by degree of adjustment for socioeconomic factors (see supplementary table S4), and a similar pattern of no difference was observed in the association of induced abortions and births with risk of endometrial cancer. Nevertheless, for type II endometrial cancers no statistically significant reduction in risk was found by each additional pregnancy. This finding could be attributable to the limited number of cases of this cancer subtype.

We carried out an analysis limited to only parous women ( $\geq 1$ birth), with uniparous women with no induced abortions as reference, to investigate the effect of number of childbirths stratified by number of induced abortions (fig 1). The results also indicate a protective effect on risk of endometrial cancer by both each additional childbirth and each additional induced abortion, in a dose-response manner.

Obesity is a strong risk factor for endometrial cancer. In a subcohort of 110567 women who had been characterised as clinically obese in hospital registers (see supplementary table S5), no difference was found in the association between pregnancy type and risk of endometrial cancer $(\mathrm{P}=0.90)$, with estimates similar to those in the complete cohort. To explore a

\begin{tabular}{|c|c|c|}
\hline \multirow[b]{2}{*}{ Pregnancy No and type } & \multicolumn{2}{|r|}{ Relative risk $(95 \% \mathrm{Cl})$} \\
\hline & Adjusted for age and period* & Adjusted for age, period, and socioeconomic factors* ${ }^{\star}$ \\
\hline \multicolumn{3}{|l|}{ First pregnancy } \\
\hline Induced abortion & $0.52(0.44$ to 0.62$)$ & $0.53(0.45$ to 0.64$)$ \\
\hline Childbirth & $0.66(0.61$ to 0.71$)$ & 0.66 (0.61 to 0.72$)$ \\
\hline \multicolumn{3}{|l|}{ Any subsequent } \\
\hline Induced abortion & $0.80(0.76$ to 0.85$)$ & $0.81(0.77$ to 0.86$)$ \\
\hline Childbirth & $0.88(0.85$ to 0.90$)$ & 0.86 (0.84 to 0.89$)$ \\
\hline \multicolumn{3}{|l|}{ Second pregnancy: } \\
\hline Induced abortion & $0.77(0.66$ to 0.90$)$ & $0.79(0.67$ to 0.92$)$ \\
\hline Childbirth & $0.85(0.79$ to 0.91$)$ & $0.83(0.77$ to 0.89$)$ \\
\hline \multicolumn{3}{|l|}{ Third pregnancy: } \\
\hline Induced abortion & $0.78(0.70$ to 0.87$)$ & $0.80(0.71$ to 0.89$)$ \\
\hline Childbirth & $0.86(0.80$ to 0.92$)$ & $0.84(0.78$ to 0.90$)$ \\
\hline \multicolumn{3}{|l|}{ Fourth pregnancy: } \\
\hline Induced abortion & $0.81(0.70$ to 0.94$)$ & $0.82(0.71$ to 0.95$)$ \\
\hline Childbirth & $0.92(0.81$ to 1.04$)$ & $0.91(0.80$ to 1.03$)$ \\
\hline \multicolumn{3}{|l|}{ Fifth pregnancy: } \\
\hline Induced abortion & 0.81 (0.63 to 1.03$)$ & $0.81(0.63$ to 1.04$)$ \\
\hline Childbirth & $0.92(0.72$ to 1.16$)$ & $0.91(0.72$ to 1.16$)$ \\
\hline \multicolumn{3}{|c|}{$\begin{array}{l}\text { *Adjusted for age, period, pregnancy history, and interaction between age and period. } \\
\text { †Additionally adjusted for educational attainment, marital status, urbanicity, and interaction between period and educational attainment, marital status, } \\
\text { and urbanicity, respectively. P value for difference between induced abortion and childbirth for risk of endometrial cancer, adjusted for age, period, } \\
\text { and socioeconomic factors, are } 0.07 \text { (relative risk } 0.53 \vee 0.66) \text { and } 0.25(0.81 v 0.86 \text { ), respectively, for first and any subsequent pregnancy. Test for } \\
\text { difference between model with different effect of each pregnancy compared with model with same effect for all pregnancies after the first pregnancy gave } \\
P=0.99 \text {. Sensitivity analysis with start of follow-up from } 20 \text { years of age presented in supplementary table } S 9 \text { gave identical results. }\end{array}$} \\
\hline
\end{tabular}




\begin{tabular}{|c|c|}
\hline Pregnancy No, type, and duration & Adjusted relative risk $(95 \% \mathrm{Cl})^{*}$ \\
\hline \multicolumn{2}{|l|}{ First pregnancyt } \\
\hline \multicolumn{2}{|l|}{ Induced abortion (weeks): } \\
\hline$\leq 5$ & 0.66 (0.30 to 1.48$)$ \\
\hline $6-7$ & $0.56(0.43$ to 0.73$)$ \\
\hline $8-9$ & $0.54(0.41$ to 0.70$)$ \\
\hline $10-11$ & $0.46(0.29$ to 0.71$)$ \\
\hline$\geq 12$ & 0.59 (0.24 to 1.41$)$ \\
\hline \multicolumn{2}{|l|}{ Childbirth (weeks): } \\
\hline$\leq 36$ & 0.61 (0.44 to 0.85$)$ \\
\hline$\geq 37$ & 0.49 (0.43 to 0.55$)$ \\
\hline \multicolumn{2}{|l|}{ Any subsequentt } \\
\hline \multicolumn{2}{|l|}{ Induced abortion (weeks): } \\
\hline$\leq 5$ & 0.71 (0.49 to 1.02$)$ \\
\hline $6-7$ & $0.75(0.68$ to 0.83$)$ \\
\hline $8-9$ & 0.84 (0.76 to 0.92$)$ \\
\hline $10-11$ & 0.93 (0.80 to 1.08$)$ \\
\hline$\geq 12$ & 0.81 (0.51 to 1.28$)$ \\
\hline \multicolumn{2}{|l|}{ Childbirth (weeks): } \\
\hline$\leq 36$ & $0.87(0.70$ to 1.10$)$ \\
\hline$\geq 37$ & 0.73 (0.69 to 0.78$)$ \\
\hline
\end{tabular}

${ }^{\star}$ Adjusted for age, period, educational attainment, marital status, urbanicity, and interaction between period and age, educational attainment, marital status, and urbanicity, respectively.

†Test for difference in fit when stratifying by gestational duration of pregnancy as in table 2, compared with stratification only by pregnancy type as in table $1, P=0.98$.

potential confounding effect of oral contraceptive use, the association between pregnancy type and risk of endometrial cancer was investigated among women from several birth cohorts, with different possible lifetime use of oral contraceptive use (see

Table 3 | Relative risk of endometrial cancer after a pregnancy compared with having one pregnancy less by pregnancy number and type, stratified by age at pregnancy, time since pregnancy, and attained age at diagnosis

Pregnancy No and type

Age at pregnancyt

Adjusted relative risk $(95 \% \mathrm{Cl})^{\star}$

First pregnancy:

\begin{tabular}{|c|c|c|}
\hline Induced abortion & 0.59 (0.48 to 0.72$)$ & $0.42(0.30$ to 0.61$)$ \\
\hline Childbirth & 0.67 (0.62 to 0.73$)$ & $0.61(0.55$ to 0.69$)$ \\
\hline \multicolumn{3}{|l|}{ Any subsequent: } \\
\hline Induced abortion & $0.90(0.80$ to 1.01$)$ & 0.78 (0.73 to 0.84$)$ \\
\hline Childbirth & 0.89 (0.86 to 0.92$)$ & 0.79 (0.75 to 0.83$)$ \\
\hline Time since pregnancy $\neq$ & $<10$ years & $\geq 10$ years \\
\hline \multicolumn{3}{|l|}{ First pregnancy: } \\
\hline Induced abortion & $0.36(0.13$ to 0.97$)$ & $0.56(0.47$ to 0.67$)$ \\
\hline Childbirth & $0.33(0.23$ to 0.50$)$ & $0.68(0.62$ to 0.73$)$ \\
\hline \multicolumn{3}{|l|}{ Any subsequent: } \\
\hline Induced abortion & $0.45(0.32$ to 0.62$)$ & 0.84 (0.79 to 0.89$)$ \\
\hline Childbirth & $0.45(0.36$ to 0.56$)$ & 0.87 (0.84 to 0.90$)$ \\
\hline Attained age§ & $<50$ years & $\geq 50$ years \\
\hline \multicolumn{3}{|l|}{ First pregnancy: } \\
\hline Induced abortion & $0.40(0.28$ to 0.56$)$ & 0.61 (0.49 to 0.75$)$ \\
\hline Childbirth & $0.42(0.35$ to 0.51$)$ & 0.73 (0.67 to 0.80$)$ \\
\hline \multicolumn{3}{|l|}{ Any subsequent: } \\
\hline Induced abortion & 0.80 (0.68 to 0.94$)$ & 0.82 (0.77 to 0.87$)$ \\
\hline Childbirth & 0.73 (0.67 to 0.81$)$ & 0.88 (0.85 to 0.91$)$ \\
\hline
\end{tabular}

*Adjusted for age, period, educational attainment, marital status, urbanicity, and interaction between period and age, educational attainment, marital status, and urbanicity, respectively. Test for difference in fit when stratifying by age at pregnancy, time since pregnancy, and attained age, respectively, compared with analysis in table 1 , $P=0.69, P=0.94$, and $P=0.13$, respectively.

†5215 events and 32745464 person years among women with a pregnancy before age 30 years, and 2462 events and 15465953 person years among women with a pregnancy at or after age 30 years.

$\ddagger 123$ events and 16813543 person years among women with less than 10 years since pregnancy, and 5681 events and 26026430 person years among women with less than 10 years since pregnancy.

$\S 929$ events and 46156519 person years among women aged less than 50 years, and 5814 events and 11191103 person years among women aged 50 or more years. supplementary table S6). No difference was found in the association by birth cohort. Furthermore, when information on spontaneous abortions available from 1977 was included (see supplementary table S7), the association between induced abortions and births and risk of endometrial cancer was not modified $(\mathrm{P}=0.08)$.

Lastly, to explore the potential effects of fecundity, we investigated the association between any pregnancy (including both induced abortions and childbirths) and risk of endometrial cancer. The protective association between pregnancy and risk of endometrial cancer was more pronounced for pregnancies at an older age (see supplementary fig S1A) and shorter time since pregnancy (see supplementary fig S1B), with any subsequent pregnancy associated with an 11\% (95\% confidence interval $6 \%$ to $15 \%$ ) risk reduction before age 25 compared with $25 \%$ (20\% to $30 \%$ ) after age 35 , and with a $52 \%$ (43\% to $60 \%)$ risk reduction within the first 10 years after pregnancy compared with a $10 \%$ ( $7 \%$ to $13 \%$ ) risk reduction after 30 or more years. In a high fecundity subcohort consisting of women with three or more pregnancies who had less than five years between their first and third pregnancy (see supplementary table S8), an additional pregnancy had a similar effect to that in other women also with three pregnancies.

\section{Discussion}

In a nationwide cohort study of pregnancy duration and risk of endometrial cancer, using information from 2.3 million women with more than 670000 induced abortions and 3.2 million childbirths, a strong protective association was found between first pregnancy and risk of endometrial cancer, with additional protection from each subsequent pregnancy. The protective association was equivalent for induced abortions and childbirths.

\section{Possible explanation for findings}

Our findings contrast with the current understanding of the cause of endometrial cancer, which is centred around the unopposed oestrogens hypothesis, whereby the risk of endometrial cancer increases with number of menstrual years (from menarche to menopause) and decreases with years of pregnancy and oral contraceptive use. ${ }^{7} 10181920$ However, a study found that each additional year of pregnancy was associated with a $22 \%$ risk reduction in risk of endometrial cancer, whereas both each additional year of oral contraceptive use and each year of delayed menarche or advanced menopause were associated with only around an $8 \%$ risk reduction, ${ }^{5}$ indicating that a pregnancy affects a woman's risk of endometrial cancer through a different mechanism than number of menstrual years.

Two underlying mechanisms could explain our finding. Foremost, the association could be due to a fecundity effect, whereby the number of pregnancies is a proxy for a woman's ability to become pregnant, and thereby a healthy endometrium and endocrine system. Several observations argue against this idea however. 


\begin{tabular}{|c|c|c|c|c|}
\hline \multirow[b]{3}{*}{ Pregnancy No and type } & \multicolumn{4}{|c|}{ Adjusted relative risk $(95 \% \mathrm{Cl}) \dagger$} \\
\hline & \multicolumn{2}{|c|}{ Cancer stage $\neq$} & \multicolumn{2}{|c|}{ Cancer subtype§ } \\
\hline & Stage I & Stage II-IV & Type I & Type II \\
\hline No of events & 2968 & 1021 & 6203 & 485 \\
\hline \multicolumn{5}{|l|}{ First pregnancy: } \\
\hline Induced abortion & $0.51(0.40$ to 0.64$)$ & 0.73 (0.53 to 1.02$)$ & 0.53 (0.44 to 0.63$)$ & $0.70(0.38$ to 1.31$)$ \\
\hline Childbirth & $0.70(0.62$ to 0.79$)$ & $0.63(0.52$ to 0.76$)$ & $0.67(0.61$ to 0.72$)$ & $0.62(0.45$ to 0.87$)$ \\
\hline \multicolumn{5}{|l|}{ Any subsequent: } \\
\hline Induced abortion & $0.86(0.80$ to 0.93$)$ & $0.80(0.70$ to 0.91$)$ & $0.81(0.76$ to 0.86$)$ & 0.84 (0.68 to 1.03$)$ \\
\hline Childbirth & $0.89(0.85$ to 0.93$)$ & 0.91 (0.84 to 0.97) & $0.84(0.82$ to 0.87$)$ & 1.09 (0.98 to 1.21$)$ \\
\hline \multicolumn{5}{|c|}{$\begin{array}{l}\text { *Analyses by competing risks between stage/subtype groups and other cancers. } \\
\text { tAdjusted for age, period, educational attainment, marital status, urbanicity, and interaction between period and age, educational attainment, marital } \\
\text { status, and urbanicity, respectively. } \\
\text { fAnalysis of cancer stage included } 19721829 \text { person years and was based on follow-up time from } 2004 \text {. Missing stage and low stage were grouped } \\
\text { together. Sensitivity analysis on cancer stage based events only after implementation of the } 2009 \mathrm{FIGO} \text { revision of stage is presented in supplementary } \\
\text { table S10. } \\
\text { \$Analysis of cancer subtype included } 57348802 \text { person years and was based on follow-up time from 1978. The few endometrial cancers not grouped in } \\
\text { type I and type II was included in the analysis as a separate outcome (see supplementary methods for definitions of endometrial cancer subtypes). }\end{array}$} \\
\hline
\end{tabular}

Firstly, we found no difference in the effect of a woman's second to fifth pregnancy, with each pregnancy having a similar protective effect, rather than a trend whereby each additional pregnancy was associated with a lower risk reduction for endometrial cancer. Secondly, when we examined the effect of an additional pregnancy in our high fecundity subcohort, the association between

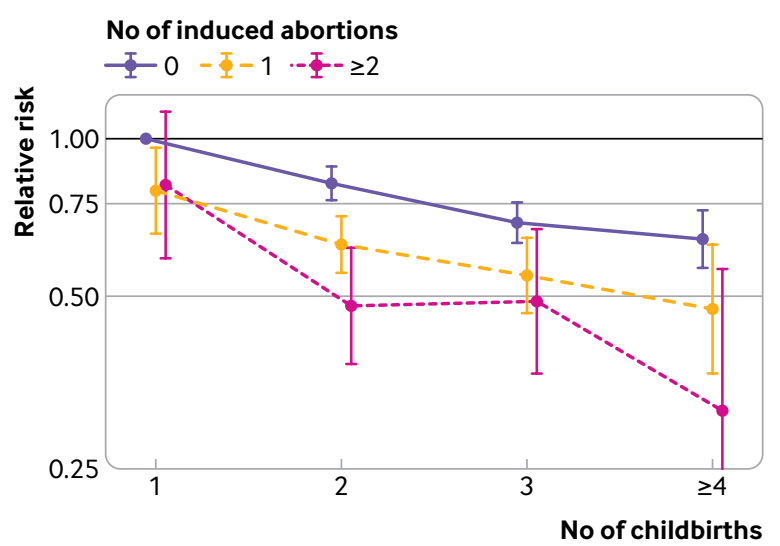

\begin{tabular}{|c|c|c|c|}
\hline $\begin{array}{l}0 \text { induced abortions } \\
\text { Events }\end{array}$ & & & \\
\hline $\begin{array}{c}1001 \\
\text { Person years (000s) }\end{array}$ & 2505 & 1006 & 332 \\
\hline 6681 & 13266 & 5012 & 1464 \\
\hline $\begin{array}{l}1 \text { induced abortion } \\
\text { Events }\end{array}$ & & & \\
\hline $\begin{array}{c}121 \\
\text { Person years (000s) }\end{array}$ & 336 & 162 & 51 \\
\hline 1388 & 2847 & 1208 & 361 \\
\hline $\begin{array}{l}\geq 2 \text { induced abortions } \\
\text { Events }\end{array}$ & & & \\
\hline $\begin{array}{c}39 \\
\text { Person years (000s) }\end{array}$ & 63 & 39 & 10 \\
\hline 528 & 956 & 459 & 160 \\
\hline
\end{tabular}

Fig 1 | Relative risk of endometrial cancer among parous women ( $\geq 1$ birth) by number of induced abortions and births, with uniparous women with no induced abortions as reference. Estimates are adjusted for age, period, educational attainment, marital status, urbanicity, and interaction between period and age, educational attainment, marital status, and urbanicity, respectively. Events and person years (in 1000s) are given for each number of induced abortion and birth. Test for interaction between induced abortions and births, $\mathrm{P}=0.92$ an additional pregnancy and risk of endometrial cancer was similar to that among other women with the same number of pregnancies. Finally, a large collaborative study from the Endometrial Cancer Consortium EC2C found a strong protective effect of parity even after adjusting for self reported infertility, ${ }^{11}$ which also argues against fecundity explaining the association between number of pregnancies and risk of endometrial cancer. Taken together, these findings point to an early gestational effect, present in the first weeks after conception, to be the most likely underlying mechanism responsible for the protective effect of pregnancy on risk of endometrial cancer.

A plausible explanation for an early gestational effect is the rapid increase of the progesterone to oestrogen ratio in the first weeks after conception, when progesterone levels increase more than threefold and oestrogen levels increase only modestly. ${ }^{21}$ In support of this hypothesis, the Norwegian Women and Cancer (NOWAC) prospective cohort study of 104318 women recently found that the risk of endometrial cancer in ever users of progesterone intrauterine devices (LNGIUS) was only $22 \%$ (95\% confidence interval $13 \%$ to $40 \%$ ) compared with never users in a multivariable analysis adjusted for oral contraceptive use, parity, body mass index, and other potential confounders. ${ }^{22}$ In addition, multiple studies have found a favourable effect of progesterone treatment for both endometrial hyperplasia-a precursor for endometrial cancer-and low stage endometrial cancer. ${ }^{23} 24$

\section{Strengths and limitations of this study}

We used a Danish nationwide cohort design with follow-up spanning four decades. Furthermore, given our access to prospectively registered clinical information on both induced abortions and births (including duration of pregnancy), we avoid recall bias, which is known to introduce biases when investigating the effects of induced abortion. ${ }^{25}$

We also investigated the effect of potential important confounders such as obesity, birth cohort, marital status, educational attainment, and urbanicity, and found no indications of confounding on the association 
between pregnancy duration and risk of endometrial cancer. However, we did not have information on lifetime use of oral contraceptives, which has been associated with a decreased risk of endometrial cancer $^{26}$ and potentially could explain the association between induced abortions and reduced risk of endometrial cancer. Nevertheless, two findings argue against this assumption. Firstly, we found no difference in the effect of induced abortion and childbirth on risk of endometrial cancer in different birth cohorts (see supplementary table S6), despite different possible lifetime use of oral contraceptives, which were available in Denmark from 1966 (see supplementary fig S2). Secondly, most of the previous studies on endometrial cancer that adjusted for oral contraceptive use found indications of a risk reducing effect of induced abortions on risk of endometrial cancer. 467910 Furthermore, our estimates of the pregnancy effect on risk of endometrial cancer were more similar when we focused on pregnancies in the same period (see supplementary table S2). This observation suggests that the marginally smaller risk reduction by births compared with induced abortions (table 1) likely is explained by confounding from oral contraceptive use, as a proportion of the births occurred before the introduction of oral contraceptives.

Spontaneous abortions were associated with less reduction in risk of endometrial cancer than induced abortions and births. However, spontaneous abortions represent a heterogeneous group of unhealthy pregnancies in contrast with induced abortions and births, which primarily are healthy pregnancies. In addition, studies report that pregnancies resulting in spontaneous abortion have low levels of gestational hormones (including progesterone), ${ }^{27}{ }^{28}$ which could explain the weaker association between spontaneous abortions and risk of endometrial cancer.

\section{Conclusions and implications}

Ourstudy shows that the protective association between pregnancy and endometrial cancer is equivalent for pregnancies resulting in induced abortion and birth, and thus suggests that the association is attributable to a woman's underlying fecundity or a biological process occurring early in gestation. The findings from our study and others support an early gestational process, as each additional pregnancy was associated with a reduction in risk of endometrial cancer, and because adjustment for infertility did not explain the effect of parity on risk. Future research is of great importance as it could lead to insights into early gestational factors that potentially could facilitate the prevention of endometrial cancer.

Contributors: $\mathrm{AH}$ conceived the study, contributed to the study design, classified register data, and performed statistical analysis, interpreted the study results, drafted the manuscript, and is the guarantor of the study. JW contributed to the study design, planned statistical analysis, oversaw the conduct of the statistical analysis, interpreted the study results, and revised the manuscript. MM contributed to the study design, interpreted the study results, and revised the manuscript. All authors had access to all of the data and take full responsibility for the integrity of the data, the accuracy of the data analysis, and the finished article. The corresponding author attests that all listed authors meet authorship criteria and that no others meeting the criteria have been omitted.

Funding: This study was supported by Helsefonden (grant No 16-B 0257 to AH), Anita og Tage Therkildsens Fond (grant No 100039 to $\mathrm{AH}$ ), and the Danish Cancer Society (grant No R167-A10791 to AH and $M M$ ). Neither funder played any role in the design or conduct of the study. All researchers acted independently from the study sponsors in all aspects of the study.

Competing interests: All authors have completed the ICMJE uniform disclosure form at www.icmje.org/coi_disclosure.pdf and declare: support from Helsefonden, Anita og Tage Therkildsens Fond, and the Danish Cancer Society; no financial relationships with any organisations that might have an interest in the submitted work in the previous three years; no other relationships or activities that could appear to have influenced the submitted work.

Ethical approval: As the study was based on deidentified information form the Danish national registers and as study participants are never contacted, consent from the Danish research bioethics committees are not required. The study's use of register data was covered by the approval from the Danish Data Protection Agency for register based studies conducted by Statens Serum Institut (approval No 2015-570102).

Data sharing: The data used in the study can be obtained by submitting a research protocol to the Danish Data Protection Agency (Datatilsynet) and, if permission is granted, by applying to the Ministry of Health's Research Service (Forskerservice) and Statistics Denmark (Danmarks Statistik) for access to the data. The data therefore do not belong to the authors and they are not permitted to share data, except in aggregate form.

The guarantor $(\mathrm{AH})$ affirms that the manuscript is an honest, accurate, and transparent account of the study being reported; that no important aspects of the study have been omitted; and that any discrepancies from the study as planned (and, if relevant, registered) have been explained.

This is an Open Access article distributed in accordance with the Creative Commons Attribution Non Commercial (CC BY-NC 4.0) license, which permits others to distribute, remix, adapt, build upon this work non-commercially, and license their derivative works on different terms, provided the original work is properly cited and the use is noncommercial. See: http://creativecommons.org/licenses/by-nc/4.0/.

1 Fitzmaurice C, Dicker D, Pain A, et al, Global Burden of Disease Cancer Collaboration. The Global Burden of Cancer 2013. JAMA Oncol 2015;1:505-27. doi:10.1001/jamaoncol.2015.0735

2 Morice P, Leary A, Creutzberg C, Abu-Rustum N, Darai E. Endometrial cancer. Lancet 2016;387:1094-108. doi:10.1016/S01406736(15)00130-0

3 Albrektsen G, Heuch I, Tretli S, Kvåle G. Is the risk of cancer of the corpus uteri reduced by a recent pregnancy? A prospective study of 765,756 Norwegian women. Int / Cancer 1995;61:485-90. doi:10.1002/ijc.2910610410

4 Brinton LA, Berman ML, Mortel R, et al. Reproductive, menstrual, and medical risk factors for endometrial cancer: results from a case-control study. Am J Obstet Gynecol 1992;167:1317-25 doi:10.1016/S0002-9378(11)91709-8

5 Dossus L, Allen N, Kaaks R, et al. Reproductive risk factors and endometrial cancer: the European Prospective Investigation into Cancer and Nutrition. Int J Cancer 2010;127:442-51. doi:10.1002/ ijc. 25050

6 Kalandidi A, Tzonou A, Lipworth L, Gamatsi I, Filippa D, Trichopoulos D. A case-control study of endometrial cancer in relation to reproductive, somatometric, and life-style variables. Oncology 1996:53:354-9. doi:10.1159/000227587

7 McPherson CP, Sellers TA, Potter JD, Bostick RM, Folsom AR. Reproductive factors and risk of endometrial cancer. The lowa Women's Health Study. Am J Epidemiol 1996;143:1195-202. doi:10.1093/oxfordjournals.aje.a008707

8 Parazzini F, Negri E, La Vecchia C, et al. Role of reproductive factors on the risk of endometrial cancer. Int J Cancer 1998;76:784-6. doi:10.1002/(SICI)1097-0215(19980610)76:6<784::AIDIJC2>3.0.CO;2-U

9 Parslov M, Lidegaard O, Klintorp S, et al. Risk factors among young women with endometrial cancer: a Danish case-control study. Am J Obstet Gynecol 2000;182:23-9. doi:10.1016/S0002 9378(00)70486-8

10 Pocobelli G, Doherty JA, Voigt LF, et al. Pregnancy history and risk of endometrial cancer. Epidemiology 2011;22:638-45. doi:10.1097/ EDE.0b013e3182263018

11 Yang HP, Cook LS, Weiderpass E, et al. Infertility and incident endometrial cancer risk: a pooled analysis from the epidemiology of 
endometrial cancer consortium (E2C2). Br J Cancer 2015;112:92533. doi:10.1038/bjc.2015.24

12 Setiawan VW, Pike MC, Karageorgi S, et al. Australian National Endometrial Cancer Study Group. Age at last birth in relation to risk of endometrial cancer: pooled analysis in the epidemiology of endometrial cancer consortium. Am J Epidemiol 2012;176:269-78. doi:10.1093/aje/kws129

13 Lohse SR, Farkas DK, Lohse N, et al. Validation of spontaneous abortion diagnoses in the Danish National Registry of Patients. Clin Epidemiol 2010;2:247-50. doi:10.2147/CLEP.S13815

14 Storm HH, Michelsen EV, Clemmensen IH, Pihl J. The Danish Cancer Registry--history, content, quality and use. Dan Med Bull 1997; 44:535-9.

15 Pecorelli S. Revised FIGO staging for carcinoma of the vulva, cervix, and endometrium. Int J Gynaecol Obstet 2009;105:103-4. doi:10.1016/j.ijgo.2009.02.012

16 Mørch LS, Kjaer SK, Keiding N, Løkkegaard E, Lidegaard Ø. The influence of hormone therapies on type I and II endometrial cancer: A nationwide cohort study. Int J Cancer 2016;138:1506-15. doi:10.1002/ijc.29878

17 Wohlfahrt J, Melbye M. Age at any birth is associated with breast cancer risk. Epidemiology 2001;12:68-73. doi:10.1097/00001648200101000-00012

18 Key TJ, Pike MC. The dose-effect relationship between 'unopposed' oestrogens and endometrial mitotic rate: its central role in explaining and predicting endometrial cancer risk. Br J Cancer 1988;57:205-12. doi:10.1038/bjc.1988.44

19 Weiderpass E, Adami H-O, Baron JA, et al. Risk of endometrial cancer following estrogen replacement with and without progestins. / Natl Cancer Inst 1999;91:1131-7. doi:10.1093/jnci/91.13.1131

20 Gavrilyuk O, Braaten T, Weiderpass E, Licaj I, Lund E. Lifetime number of years of menstruation as a risk index for postmenopausal endometrial cancer in the Norwegian Women and Cancer Study. Acto Obstet Gynecol Scand 2018;97:1168-77. doi:10.1111/aogs.13381

21 Liu J. Endocrinology of Pregnancy. In: Creasy RK, Resnik R, lams ID, Lockwood C), Moore TR, Greene MF, eds. Creasy and Resnik's Maternal-Fetal Medicine: Principles and Practice. 7th ed. Saunders, 2014: 100-11.
22 Jareid M, Thalabard J-C, Aarflot M, Bøvelstad HM, Lund E, Braaten T. Levonorgestrel-releasing intrauterine system use is associated with a decreased risk of ovarian and endometrial cancer, without increased risk of breast cancer. Results from the NOWAC Study. Gynecol Oncol 2018;149:127-32. doi:10.1016/j.ygyno.2018.02.006

23 Gunderson CC, Fader AN, Carson KA, Bristow RE. Oncologic and reproductive outcomes with progestin therapy in women with endometrial hyperplasia and grade 1 adenocarcinoma: a systematic review. Gynecol Oncol 2012;125:477-82. doi:10.1016/j. ygyno.2012.01.003

$24 \varnothing$ ørbo A, Vereide A, Arnes M, Pettersen I, Straume B. Levonorgestrelimpregnated intrauterine device as treatment for endometrial hyperplasia: a national multicentre randomised trial. BJOG 2014;121:477-86. doi:10.1111/1471-0528.12499

25 Lindefors-Harris B-M, Eklund G, Adami H-O, Meirik O. Response bias in a case-control study: analysis utilizing comparative data concerning legal abortions from two independent Swedish studies. Am J Epidemiol 1991;134:1003-8. doi:10.1093/oxfordjournals.aje. a116173

26 Collaborative Group on Epidemiological Studies on Endometrial Cancer. Endometrial cancer and oral contraceptives: an individual participant meta-analysis of 27276 women with endometrial cance from 36 epidemiological studies. Lancet Oncol 2015;16:1061-70. doi:10.1016/S1470-2045(15)00212-0

27 Johnson MR, Riddle AF, Sharma V, Collins WP, Nicolaides KH, Grudzinskas JG. Placental and ovarian hormones in anembryonic pregnancy. Hum Reprod 1993;8:112-5. doi:10.1093/oxfordjournals. humrep.a137857

28 Whittaker PG, Schreiber CA, Sammel MD. Gestational hormone trajectories and early pregnancy failure: a reassessment. Reprod Biol Endocrinol 2018;16:95. doi:10.1186/s12958-018-0415-1

Supplementary information: additional information on methods and materials, demographic information, in addition to sensitivity analyses 